\title{
Point-of-care cerebrospinal fluid Gram stain for the management of acute meningitis in adults: a retrospective observational study
}

Tomohiro Taniguchi ${ }^{1,2^{*}} \mathbb{0}$, Sanefumi Tsuha ${ }^{1,3}$, Soichi Shiiki ${ }^{1}$ and Masashi Narita ${ }^{1}$

\begin{abstract}
Background: Gram stain of cerebrospinal fluid (CSF) is widely used in the diagnosis of acute meningitis, however, it is often conducted in the laboratory, as only some hospitals have access to point-of-care Gram stain (PCGS). The purpose of this study was to demonstrate the clinical impact and utility of PCGS in diagnosing and treating both bacterial and aseptic meningitis in adults.
\end{abstract}

Methods: This was a hospital-based, retrospective observational study at a referral center in Okinawa, Japan. We reviewed the records of all patients aged 15 years or older who were admitted to the Division of Infectious Diseases between 1995 and 2015 and finally diagnosed with bacterial $(n=34)$ or aseptic meningitis $(n=97)$. For bacterial meningitis, we compared the treatments that were actually selected based on PCGS with simulated treatments that would have been based on the Japanese guidelines. For aseptic meningitis, we compared the rates of antibiotic use between real cases where PCGS was available and real cases where it was not.

Results: PCGS was the most precise predictor for differentiating between bacterial and aseptic meningitis (sensitivity 91.2\%, specificity 98.9\%), being superior in this regard to medical histories, vital signs and physical examinations, and laboratory data available in the emergency room (ER). In bacterial meningitis, PCGS reduced the frequency of meropenem use $(1 / 34=3.0 \%)$ compared with simulated cases in which PCGS was not available $(19 / 34=55.9 \%)(p<0.001)$. In aseptic meningitis cases, the rate of antibiotic administration was lower when PCGS was used (38/97=39.2\%) than when it was not $(45 / 74=60.8 \%)(p=0.006)$.

Conclusions: PCGS of CSF distinguishes between bacterial and aseptic meningitis more accurately than other predictors available in the ER. Patients with bacterial meningitis are more likely to receive narrower-spectrum antimicrobials when PCGS is used than when it is not. PCGS of CSF thus can potentially suppress the empiric use of antimicrobials for aseptic meningitis.

Keywords: Point-of-care gram stain, Cerebrospinal fluid, Acute meningitis, Bacterial meningitis, Suppurative meningitis, Aseptic meningitis

${ }^{*}$ Correspondence: taniyan260566@gmail.com

1 Division of Infectious Diseases, Department of Internal Medicine, Okinawa Chubu Hospital, 281 Miyazato, Uruma, Okinawa 904-2293, Japan

Full list of author information is available at the end of the article

\begin{abstract}
Background
Rapid diagnosis of bacterial meningitis and early antimicrobial treatment are essential for a favorable outcome [1-3], and empiric broad-spectrum antibiotics should be given as soon as possible to patients with suspected bacterial meningitis. At the same time, these broad-spectrum antibiotics should not be administered if not needed: antimicrobial-resistant meningitis,
\end{abstract}

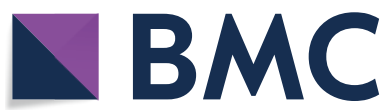

(c) The Author(s) 2020. This article is licensed under a Creative Commons Attribution 4.0 International License, which permits use, sharing, adaptation, distribution and reproduction in any medium or format, as long as you give appropriate credit to the original author(s) and the source, provide a link to the Creative Commons licence, and indicate if changes were made. The images or other third party material in this article are included in the article's Creative Commons licence, unless indicated otherwise in a credit line to the material. If material is not included in the article's Creative Commons licence and your intended use is not permitted by statutory regulation or exceeds the permitted use, you will need to obtain permission directly from the copyright holder. To view a copy of this licence, visit http://creativeco mmons.org/licenses/by/4.0/. The Creative Commons Public Domain Dedication waiver (http://creativecommons.org/publicdomain/ zero/1.0/) applies to the data made available in this article, unless otherwise stated in a credit line to the data. 
including meropenem-resistant Streptococcus pneumoniae in the CSF, has already been isolated, and its spread in Japan and Taiwan is a cause for concern [4, 5]. Distinguishing bacterial meningitis from aseptic meningitis, which is caused mainly by viral infection, is therefore crucial, but is challenging because the symptoms are often similar $[3,6]$. There is no single laboratory test that adequately discriminates between bacterial and aseptic meningitis [7, 8]. Due to this diagnostic uncertainty, most patients with aseptic meningitis are unnecessarily treated with broad-spectrum antibiotics [3].

Gram stain of the cerebrospinal fluid (CSF) is a rapid, inexpensive, and accurate method for detecting the etiologic agents of bacterial meningitis $[6,9]$. The sensitivity of CSF Gram staining is $60-98 \%$, depending on the patient population, type of bacteria, and previous antimicrobial treatment, and its specificity is nearly 100\% [9-17]. Current guidelines from the Infectious Diseases Society of America recommend Gram staining of the CSF for all suspected meningitis patients [8]; in practice, however, this is only possible at the select institutions where Gram stain examination by a microbiology technician is available on demand at any time. At institutions where Gram staining is not available around the clock, empiric antibiotics are started in $60.8 \%(45 / 74)$ of aseptic meningitis cases in adults [11].

At Okinawa Chubu Hospital in Japan, point-of-care Gram stain (PCGS) has been used routinely for the diagnosis of infectious diseases since 1976 [18]. As PCGS can be performed by clinicians in the emergency room (ER) at any time, our physicians rely on PCGS to help them choose appropriate antibiotics. However, the utility of PCGS in treating acute meningitis has never been evaluated, and its clinical impact remains unclear.

The objective of this study was to clarify whether PCGS performed by clinicians is a valuable tool for the diagnosis and treatment of bacterial and aseptic meningitis in adults. For that purpose, we evaluated whether PCGS can discriminate between bacterial and aseptic meningitis, whether the selection of targeted therapies based on PCGS results as opposed to reliance on the Japanese guidelines alone can suppress broad-spectrum antimicrobial use for bacterial meningitis, and whether PCGS as opposed to no Gram stain findings can limit the use of empiric antibiotics for aseptic meningitis. Our findings lead us to recommend PCGS to ensure a more precise diagnosis of acute meningitis and to reduce the overuse of broadspectrum antimicrobial agents for both bacterial and aseptic meningitis.

\section{Methods}

\section{Study design and setting}

This was a hospital-based, retrospective observational study. The study setting was Okinawa Chubu Hospital, located in central Okinawa, a subtropical region of Japan. Approximately 39,000 patients visit the ER annually and nearly 7,000 patients are hospitalized through the ER each year [19]. Most patients who are suspected of meningitis are initially worked up at the ER. Adolescent and adult patients who are diagnosed with bacterial or aseptic meningitis are admitted to the Division of Infectious Diseases, while patients with meningitis related to surgical interventions such as ventricular peritoneal shunt infection are admitted to the Division of Neurosurgery and patients with encephalitis are admitted to the Division of Neurology.

\section{Case definitions and data collection}

Bacterial meningitis was defined as clinically evident acute meningitis and positive routine bacterial culture of CSF, regardless of whether antibiotics were administered before the first CSF sample was obtained. This definition was used because bacterial culture is still regarded as the gold standard for the diagnosis of bacterial meningitis $[20,21]$. Aseptic meningitis was defined as clinically evident acute meningitis with pleocytosis in the CSF and no growth on routine bacterial culture of the CSF $[16,22]$.

In this study, all clinical information of acute meningitis was collected from hospital database between January 1995 and January 2015. The inclusion criteria were (1) all patients aged 15 years or older who were (2) admitted to the Division of Infectious Diseases and who were (3) finally diagnosed with bacterial or aseptic meningitis. The exclusion criteria were (1) bacterial meningitis in which CSF culture was negative, a situation that was typically caused by prior antimicrobial exposure, which we chose to exclude because this study was intended to investigate culture-proven bacterial meningitis; (2) certain pathogens such as Bartonella, Leptospira, and Mycobacterium tuberculosis that were not incubated in routine CSF cultures; (3) aseptic meningitis in which prior antimicrobial exposure was positive within $48 \mathrm{~h}$ before the first CSF sample was obtained and also in which empiric antimicrobials were started after CSF sampling (this qualification was intended to exclude false culturenegative bacterial meningitis); (4) aseptic meningitis caused by non-infectious diseases such as Sjögren's syndrome, which we chose to exclude because this study was focused on infectious meningitis; (5) all cases in human immunodeficiency virus (HIV)-infected patients, which we chose to exclude because their opportunistic infections and clinical courses are quite different from those in cases of community-acquired meningitis; (6) meningitis 
cases that had been diagnosed at a previous hospital and referred to our hospital; and (7) cases whose CSF data was missing.

\section{CSF examinations}

CSF specimens obtained in the ER were immediately transported to a laboratory where conventional tests including cell counts, glucose and protein levels were measured for every patient. Each sample was first centrifuged at $1500 \mathrm{rpm}$ for $5 \mathrm{~min}$. As soon as the supernatant had been discarded, the sediment was returned to the ER for PCGS. All CSF sediment samples were placed on glass slides, fixed with flame or hot air, and Gram stained with Barmii M manufactured by Muto Pure Chemicals (Tokyo, Japan). We used a four-step of dying procedure using crystal violet, $2 \%$ iodine sodium hydroxide, acetone ethyl alcohol, and $0.1 \%$ fuchsine. The slides were examined in the ER by in-house staff members including trained resident physicians (postgraduate years 1 and 2) to identify each etiologic agent and select an appropriate targeted antimicrobial therapy $[18,23]$. Staff members examined the slides under conventional light microscopy at $100 \times$ magnification, then at $1000 \times$ magnification under immersion oil to semi-quantify CSF polymorphonuclear neutrophils or mononuclear lymphocytes and to search for bacteria or yeasts. All findings were confirmed by senior residents (postgraduate years 3 to 5 ) and attending physicians. Whenever a PCGS result was unclear, our physicians consulted a staff microbiologist, although this option was only available during daytime hours. Otherwise, they evaluated the PCGS results themselves.

In the laboratory, CSF samples were subjected to Gram stain, Ziehl-Neelsen stain, Indian ink stain, and cultures for the detection of etiologic agents. With regard to the Gram stain procedure, only the solutions were different from those usually used for PCGS. The laboratory technicians used Favor G manufactured by Nissui Pharmaceutical (Tokyo, Japan). This was a three-step staining procedure: $0.2 \%$ Victoria blue, $2 \%$ picric acid alcohol, and $0.04 \%$ fuchsine.

\section{PCGS and selection of antimicrobial agents for bacterial meningitis}

In Gram staining of the CSF, Gram-positive diplococci or cocci in chains suggested Streptococcus pneumoniae, Streptococcus spp., Enterococcus spp., or other Grampositives including Lactococcus. In such cases, a thirdgeneration cephalosporin (cefotaxime or ceftriaxone) and vancomycin was frequently selected given the high proportion of penicillin-resistant S. pneumoniae of the CSF in Japan [24]. If there was a possibility of Enterococcus infection, ampicillin or penicillin $\mathrm{G}$ was combined with these agents. Gram-negative rods suggested Enterobacteriaceae such as Klebsiella pneumoniae or Escherichia coli, or other Gram-negatives including Pseudomonas aeruginosa; in such cases a third-generation cephalosporin (cefotaxime, ceftriaxone, or ceftazidime) was chosen. If an extended-spectrum beta-lactamase (ESBL)-producing bacterial strain was suspected based on previous culture findings, meropenem was selected. Gram-positive small rods suggested Listeria monocytogenes, in which case ampicillin was used. Gram-negative coccobacilli suggested Haemophilus influenzae. Gram-negative diplococci suggested Neisseria meningitidis.

In the simulated portion of our study, we reviewed all findings other than Gram stain findings from the same 34 bacterial meningitis patients and selected a course of treatment according to the current Japanese guidelines. When the guidelines recommended empiric antimicrobial therapy, we referred to the Practical Guideline for Bacterial Meningitis 2014 (PGBM), published by Societas Neurologica Japonica, the Japanese Society of Neurological Therapeutics and the Japanese Society for Neuroinfectious Diseases [24], to determine which antimicrobial agent would be used. If patients had debilitating associated diseases or immunodeficiency, the PGBM recommended ceftazidime ( $2 \mathrm{~g} \mathrm{q} 8 \mathrm{~h}$ or $6 \mathrm{~g} /$ day) plus vancomycin (30-60 $\mathrm{mg} / \mathrm{kg} /$ day or $3 \mathrm{~g} /$ day) plus ampicillin $(2 \mathrm{~g} \mathrm{q} 4 \mathrm{~h}$ or $12 \mathrm{~g} /$ day), or meropenem ( $2 \mathrm{~g} \mathrm{q} 8 \mathrm{~h}$ or $6 \mathrm{~g} /$ day) plus vancomycin. If patients were immunocompetent and were over 50 years of age, a third-generation cephalosporin (cefotaxime $2 \mathrm{~g}$ q4-6 h or 8-12 g/day, or ceftriaxone $2 \mathrm{~g} \mathrm{q} 12 \mathrm{~h}$ or $4 \mathrm{~g} /$ day) plus vancomycin plus ampicillin, or meropenem plus vancomycin, was recommended. Otherwise, the PGBM recommended meropenem.

For each bacterial meningitis patient, we compared the targeted therapy that was actually selected based on PCGS findings with the empirical therapy that would have been recommended by the PGBM in the simulation excluding PCGS findings. As part of this comparison, we analyzed the first-choice antimicrobials in terms of their dosage and cost, i.e., their original pharmaceutical price in Japanese yen, which is determined by the Ministry of Health, Labour and Welfare, as of the first day of admission.

\section{PCGS and rates of empiric antibiotic use in aseptic meningitis}

To assess the utility of PCGS in selecting optimal treatments for aseptic meningitis patients, we compared the rate of empiric antibiotic use in our aseptic meningitis patients, all of whom were known to have negative Gram stain results, with the rate of antibiotic use in a previous study in which Gram staining findings were not available [11]. 


\section{Outcome measures}

Primary outcomes were the sensitivity and specificity of PCGS for the differential diagnosis between bacterial and aseptic meningitis, the rate of meropenem administration among bacterial meningitis patients, and the rate of all antibiotic administration excluding antiviral agents among aseptic meningitis patients. Meropenem was selected for particular study because it has broad-spectrum coverage and is highly recommended as an empiric treatment by the Japanese guidelines as stated in the PGBM. Vancomycin is also broad-spectrum but is not suitable as an outcome measure because drug-resistant $S$. pneumoniae in the CSF is common in Japan, and the co-administration of vancomycin is necessary even if $S$. pneumoniae is suspected by PCGS. Secondary outcomes were the accordance between PCGS and CSF culture results; the effectiveness, as assessed by drug susceptibility tests on CSF cultures, of the antibiotics chosen based on PCGS findings; and the total drug cost on the first day of admission.

\section{Statistical analysis}

To ensure an adequate sample size of bacterial meningitis patients, we initially planned to collect at least 30 patients, which would give us the minimum statistical power necessary to calculate sensitivity and specificity. We assumed that the rate of meropenem administration in the simulated PGBM-based portion of the study would be around 0.5, because the PGBM recommends the empiric use of meropenem or a third-generation cephalosporin for both immunocompromised and immunocompetent patients. In the PCGS group, in contrast, we expected a much lower rate of meropenem administration. Therefore, assuming that the rate of meropenem use would be 0.5 in the guideline group and 0.2 in the PCGS group, and assuming a guideline-group to PCGS-group ratio of 1:1, 80\% power, and a one-sided alpha level of 0.05 , we calculated that 31 patients per group would be needed.

Among aseptic meningitis patients, we hypothesized that a $20 \%$ difference in the rates of antibiotic administration between the PCGS and non-PCGS groups would be clinically meaningful. In a study in Switzerland where round-the-clock access to Gram staining was not available [11], the rate of empiric antimicrobial use in adults with aseptic meningitis was $0.6(45 / 74)$. To detect a rate of empiric antibiotic use of 0.4 in the PCGS group, therefore, assuming $80 \%$ power and a one-sided alpha level of 0.05 , we estimated that at least 79 patients would be needed.

The $\chi^{2}$ test or Fisher's exact test was used for categorical variables, and Student's $t$-test was used for numerical variables. The receiver operating characteristic (ROC) curve was analyzed to determine the best threshold for each variable to discriminate between bacterial and aseptic meningitis. The sensitivity, specificity, accuracy, positive and negative likelihood ratios, and areas under the ROC curve with 95\% confidence intervals were calculated. Kappa statistics were also used to evaluate the agreement between the results of PCGS and those of CSF cultures. These results were calculated using Stata software (version 16.1; StataCorp, College Station, TX, USA).

\section{Results}

Thirty-four patients with bacterial meningitis and 97 patients with aseptic meningitis were enrolled. Table 1 shows a comparison of bacterial and aseptic meningitis cases. Patient age ranged from 22 to 91 in bacterial meningitis cases and from 15 to 76 in aseptic meningitis cases.

In twelve patients with bacterial meningitis, antibiotics had previously been prescribed; these were amoxicillin, amoxicillin/clavulanic acid, piperacillin, cefotiam (2nd generation cephalosporin), cefmetazole, cefotaxime, ceftriaxone, cefoperazone/sulbactam, clarithromycin, ofloxacin and levofloxacin. No antiviral agents were prescribed. In nine patients with aseptic meningitis, penicillin G, amoxicillin/clavulanic acid, cefalexin, clarithromycin, fleroxacin, levofloxacin, acyclovir, or oseltamivir had been administered within $48 \mathrm{~h}$ prior to arrival.

There was only one patient with pneumococcal meningitis whose CSF cell count was 0 . This patient's CSF protein was not elevated $(48 \mathrm{mg} / \mathrm{dL})$ and CSF glucose was decreased $(49 \mathrm{mg} / \mathrm{dL})$ relative to blood sugar $(127 \mathrm{mg} /$ dL). Gram-positive diplococci were detected with PCGS.

S. bovis was reclassified into three different biotypes in 2003 [25]. Among the four S. bovis cases, only one could be more specifically identified as Streptococcus infantarius subsp. coli. The other three cases were of unidentified subtype because they were old cases from before the subtypes were defined. The five cases in which CSF cultures were positive but blood cultures were negative were two S. pneumoniae, one $H$. influenzae, one $K$. pneumoniae, and one S. bovis infection. Only one patient was infected with two bacterial species: Bacteroides fragilis was cultured from the CSF, and both B. fragilis and E. coli were cultured from the blood. No ESBL-producing bacteria were found in this study.

In the aseptic meningitis cases, mumps, Varicella zoster, Herpes simplex, and cytomegalovirus were identified as the etiologic agents either clinically or through serologic tests or polymerase chain reactions, but not through viral cultures.

Among the bacterial meningitis cases, the point of entry varied: four cases were suspected to have arisen 
Table 1 Comparison between bacterial and aseptic meningitis

\begin{tabular}{|c|c|c|c|c|c|}
\hline & $\begin{array}{l}\text { Bacterial meningitis } \\
\mathrm{N}=34\end{array}$ & & $\begin{array}{l}\text { Aseptic meningitis } \\
\mathrm{N}=97\end{array}$ & & $p$ value \\
\hline Median age (IQR) & $60(44-68)$ & & $30(23-34)$ & & $<0.0001^{*}$ \\
\hline Male sex & 19/34 (55.9\%) & & $51 / 97(52.6 \%)$ & & 0.74 \\
\hline \multicolumn{6}{|l|}{ Medical histories } \\
\hline Symptom duration days (IQR) & $2.5(0-5)$ & & $3(1-4)$ & & 0.71 \\
\hline Shaking chills & $9 / 34(26.5 \%)$ & & $6 / 97(6.2 \%)$ & & $0.001^{*}$ \\
\hline Previous antibiotic exposure within $48 \mathrm{~h}$ & $12 / 34(35.3 \%)$ & & 9/97 (9.2\%) & & $<0.001^{*}$ \\
\hline Immunocompromised host & $17 / 34(50.0 \%)$ & & $8 / 97(8.2 \%)$ & & $<0.001^{*}$ \\
\hline \multicolumn{6}{|l|}{ Vital signs and physical examinations } \\
\hline Systolic blood pressure (mmHg) (IQR) & $136(120-150)$ & & $112(100-120)$ & & $<0.0001^{*}$ \\
\hline Pulse rate (/min) (IQR) & $113(100-126)$ & & $88(80-96)$ & & $<0.0001^{*}$ \\
\hline Respiratory rate (/min) (IQR) & $24(20-30)$ & & $20(18-22)$ & & $0.0002^{*}$ \\
\hline Body temperature $\left({ }^{\circ} \mathrm{C}\right)(\mathrm{IQR})$ & $38.6(37.9-39.3)$ & & $37.7(37.1-38.4)$ & & $<0.0001^{*}$ \\
\hline Glasgow Coma Scale (IQR) & $11(9-14)$ & & $15(15-15)$ & & $<0.0001^{*}$ \\
\hline Neck stiffness & 28/33 (84.9\%) & & $47 / 95(49.5 \%)$ & & $0.002^{*}$ \\
\hline Jolt accentuation & $5 / 6(83.3 \%)$ & & $53 / 64(82.8 \%)$ & & 0.97 \\
\hline \multicolumn{6}{|l|}{ Laboratory data } \\
\hline 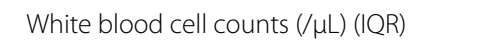 & $13,800(11,000-16,200)$ & & 7800 (5900-9900) & & $<0.0001^{*}$ \\
\hline C-reactive protein (mg/dL) (IQR) & $6.7(1.3-19.4)$ & & $0.18(0.02-0.9)$ & & $<0.0001^{*}$ \\
\hline CSF cell counts $(/ \mu \mathrm{L})(\mathrm{IQR})$ & $1116(140-4200)$ & & $80(37-156)$ & & $<0.0001^{*}$ \\
\hline CSF monocytes (\%) (IQR) & $5(3-17)$ & & $74(27-95)$ & & $<0.0001^{*}$ \\
\hline CSF protein (mg/dL) (IQR) & $287(100-420)$ & & $66(51-94)$ & & $<0.0001^{*}$ \\
\hline CSF glucose (mg/dL) (IQR) & $37(1-59)$ & & $57(53-63)$ & & $0.0001^{*}$ \\
\hline CSF glucose/blood glucose ratio (IQR) & $0.17(0.006-0.43)$ & & $0.55(0.50-0.60)$ & & $<0.0001^{*}$ \\
\hline CSF bacteria confirmed with PCGS & $31 / 34(91.2 \%)$ & & $1 / 97(1.0 \%)$ & & $<0.001^{*}$ \\
\hline CSF culture positivity & $34 / 34(100 \%)$ & & 0 & & $<0.001^{*}$ \\
\hline Blood culture positivity & 29/34 (85.3\%) & & 0 & & $<0.001^{*}$ \\
\hline \multirow[t]{8}{*}{ Etiologic agent } & Streptococcus pneumoniae & 20 & Mumps virus & 8 & \\
\hline & Klebsiella pneumoniae & 5 & Varicella zoster & 5 & \\
\hline & Streptococcus bovis & 4 & Cytomegalovirus & 2 & \\
\hline & Bacteroides fragilis & 1 & Herpes simplex & 2 & \\
\hline & Escherichia coli & 1 & Unknown & 80 & \\
\hline & Haemophilus influenzae & 1 & & & \\
\hline & Lactococcus lactis cremoris & 1 & & & \\
\hline & Listeria monocytogenes & 1 & & & \\
\hline \multicolumn{6}{|l|}{ Complications } \\
\hline Infection sites other than meningitis & $9 / 34(26.7 \%)$ & & 13/97 (13.4\%) & & 0.079 \\
\hline Strongyloides coinfection & 10/34 (29.4\%) & & 0 & & $<0.001^{*}$ \\
\hline \multicolumn{6}{|l|}{ Treatment } \\
\hline Antibiotics other than acyclovir & $34 / 34(100 \%)$ & & $38 / 97(39.2 \%)$ & & $<0.001^{*}$ \\
\hline Steroid use & $4 / 34(11.8 \%)$ & & 0 & & $0.005^{*}$ \\
\hline Antimicrobial treatment days (IQR) & $14(12-14)$ & & $3(3-5)$ & & $0.0001^{*}$ \\
\hline \multicolumn{6}{|l|}{ Outcome } \\
\hline Death & $4 / 34(11.8 \%)$ & & 0 & & $0.004^{*}$ \\
\hline Neurological sequelae & 4/30 (13.3\%) & & 0 & & $0.004^{*}$ \\
\hline
\end{tabular}

CSF cerebrospinal fluid, IQR interquartile range, $P C G S$ point-of-care Gram stain ${ }^{*} p<0.05$ 
through contiguous spread from local infection: these were three cases of otitis media and one case of sinusitis. The remaining seven cases were suspected to have emerged from distant foci of infection: these were three cases of pneumonia, two cases of septic arthritis, and one case each of infective endocarditis and osteomyelitis. Both septic arthritis cases were co-infected: one with infective endocarditis and one with osteomyelitis. Thus, nine patients had other simultaneous infections. Among the aseptic meningitis patients, thirteen cases had simultaneous other infections: eight of these were mumps in the parotid gland, four were skin lesions of Varicella zoster, and one was Herpes simplex virus in the genital area.

Strongyloides stercoralis coinfection was determined based on the presence of $S$. stercoralis larvae or eggs in the stool, gastric juice or sputum, or on previous episodes of proven strongyloidiasis associated with repeated meningitis. Ivermectin or thiabendazole was used with antimicrobials to treat $S$. stercoralis coinfection.

Among the 38 aseptic meningitis cases treated with empirical antimicrobial treatments, the antimicrobial agents were as follows: 19 ceftriaxone, 15 cefotaxime, 6 ampicillin, 3 vancomycin, and 1 doxycycline. Doxycycline was empirically chosen for possible leptospirosis, although the possibility of leptospirosis was later disproven. More than one antimicrobial was used simultaneously in several cases. In addition, acyclovir was empirically used in 10 patients for possible herpes infection.

Four bacterial meningitis patients survived with neurological sequelae; these included one with both sensory hearing loss and facial palsy, and one each with sensory hearing loss, neurogenic bladder, and impaired consciousness. In all of these, the etiologic agent was $S$. pneumoniae. Dexamethasone was administered in one case but not in the others.

Four patients died because of bacterial meningitis. In one of them, small Gram-negative rods were observed in PCGS, and ceftazidime and clindamycin were chosen accordingly. In this case, Bacteroides fragilis was cultured from the CSF, and both B. fragilis and E. coli were cultured from the blood.

Table 2 compares the effectiveness of PCGS with that of other variables for differentiating between bacterial and aseptic meningitis. Among the age and history variables, age over 45 had the highest area under the ROC curve (AUC). Among the vital signs and physical examination variables, Glasgow Coma Scale (GCS) less than 15 had the highest AUC. Among PCGS and laboratory data, PCGS had the highest AUC. Among all variables, PCGS was the most accurate predictor. When age was less than 45, GCS was 15, and PCGS was negative, $98.8 \%(84 / 85)$ of cases were aseptic meningitis.

Table 2 Effectiveness of different variables at correctly predicting a diagnosis of bacterial vs. aseptic meningitis

\begin{tabular}{|c|c|c|c|c|c|c|}
\hline & Sensitivity & Specificity & Accuracy & $\mathrm{LR}+$ & LR- & AUC $(95 \% \mathrm{Cl})$ \\
\hline \multicolumn{7}{|l|}{ Age and history } \\
\hline Age $\geq 45$ & 73.5 & 89.7 & 85.5 & 7.1 & 0.30 & $0.82(0.73-0.90)$ \\
\hline Immunocompromised host & 50.0 & 91.2 & 80.9 & 6.1 & 0.54 & $0.71(0.62-0.80)$ \\
\hline Shaking chills & 26.5 & 93.8 & 76.3 & 4.3 & 0.78 & $0.60(0.52-0.68)$ \\
\hline \multicolumn{7}{|l|}{ Vital signs and physical examinations } \\
\hline Glasgow Coma Scale < 15 & 81.2 & 97.9 & 93.7 & 38.2 & 0.19 & $0.90(0.83-0.97)$ \\
\hline Pulse rate $\geq 100(/ \mathrm{min})$ & 76.4 & 80.0 & 79.1 & 3.8 & 0.29 & $0.78(0.70-0.86)$ \\
\hline Systolic BP $\geq 120(\mathrm{mmHg})$ & 85.3 & 61.1 & 67.4 & 2.2 & 0.24 & $0.73(0.65-0.81)$ \\
\hline Respiratory rate $\geq 22(/ \mathrm{min})$ & 64.7 & 73.9 & 71.4 & 2.5 & 0.48 & $0.69(0.60-0.79)$ \\
\hline Neck stiffness & 84.9 & 50.5 & 59.4 & 1.7 & 0.30 & $0.68(0.60-0.76)$ \\
\hline Body temperature $\geq 38.0\left({ }^{\circ} \mathrm{C}\right)$ & 69.7 & 61.1 & 63.3 & 1.8 & 0.50 & $0.65(0.56-0.75)$ \\
\hline \multicolumn{7}{|l|}{ PCGS and laboratory data } \\
\hline CSF PCGS & 91.2 & 99.0 & 97.0 & 88.4 & 0.09 & $0.95(0.90-1.00)$ \\
\hline CSF monocyte $\leq 20(\%)$ & 85.3 & 83.3 & 83.9 & 5.1 & 0.18 & $0.84(0.77-0.91)$ \\
\hline CSF glucose/blood glucose ratio $\leq 0.5$ & 85.3 & 75.5 & 78.1 & 3.5 & 0.20 & $0.80(0.73-0.88)$ \\
\hline $\mathrm{WBC} \geq 10,000(/ \mu \mathrm{L})$ & 78.8 & 76.8 & 77.3 & 3.4 & 0.28 & $0.78(0.70-0.86)$ \\
\hline CSF protein $\geq 100(\mathrm{mg} / \mathrm{dL})$ & 76.5 & 77.3 & 77.1 & 3.4 & 0.30 & $0.77(0.69-0.85)$ \\
\hline $\mathrm{CRP} \geq 1.0(\mathrm{mg} / \mathrm{dL})$ & 77.4 & 76.0 & 76.4 & 3.2 & 0.30 & $0.77(0.68-0.85)$ \\
\hline CSF cell $\geq 100(/ \mu \mathrm{L})$ & 76.5 & 58.8 & 63.4 & 1.9 & 0.40 & $0.68(0.59-0.76)$ \\
\hline
\end{tabular}

AUC area under curve, $B P$ blood pressure, $C l$ confidence interval, CRP C-reactive protein, CSF cerebrospinal fluid, GCS Glasgow Coma Scale, LR likelihood ratio, $P C G S$ point-of-care Gram stain, WBC white blood cell 
Table 3 shows the concordance between PCGS and CSF cultures. The kappa coefficient was 0.903 (95\% Confidence Interval $0.887-0.906$ ). The five cases whose PCGS results did not match their CSF culture results were one false-positive, three false-negatives, and one morphological mismatch. None of them had previous antimicrobial administration. In the one false-positive case, Gram-positive coccus was detected through PCGS but not cultured. The number of Gram-positive cocci in this case was low. Gram-positives were not confirmed through Gram stain in the laboratory. In one of the falsenegatives, no bacteria were observed through PCGS, but a Gram-positive rod species identified as $L$. monocytogenes was cultured. This patient was an alcohol abuser. In another false-negative, no bacteria was observed through PCGS, but S. pneumoniae was cultured. This patient also had septic arthritis of the knee and infective endocarditis. In the third false-negative, no bacteria was confirmed through PCGS or Gram staining in the laboratory, but a Gram-negative rod identified as $H$. influenzae

Table 3 Concordance between point-of-care Gram stain and CSF culture results

\begin{tabular}{lllll}
\hline \multicolumn{5}{c}{ CSF culture } \\
\cline { 2 - 5 } & GPC & GNR & GPR & Negative \\
\hline Point-of-care Gram stain & & 0 & 1 \\
GPC & 23 & 0 & 0 & 0 \\
GNR & 1 & 7 & 0 & 0 \\
GPR & 0 & 0 & $1(\mathrm{Lm})$ & 87 \\
Negative & $1(\mathrm{Sp})$ & $1(\mathrm{Hi})$ &
\end{tabular}

CSF cerebrospinal fluid, GNR Gram-negative rod, GPC Gram-positive coccus, GPR Gram-positive rod, Hi Haemophilus influenzae, Lm Listeria monocytogenes, $S p$ Streptococcus pneumoniae

Kappa coefficient 0.903 (95\% Cl 0.887-0.906) was cultured. The blood culture was also negative. This patient had a past medical history of CSF leakage. In the morphological mismatch case, Gram-negative coccobacilli were suspected through PCGS. However, a physician consulted the laboratory, and they revealed Gram-positive diplococci. This case was the second incidence of CSF leakage in a patient who had had $H$. influenzae infection previously.

Table 4 compares the targeted antimicrobial agent that was chosen based on PCGS results with the agent that would have been chosen in the same situation in the absence of PCGS data according to the current Japanese guidelines, i.e., the simulated empirical agent. When PCGS data was used, third-generation cephalosporins (cefotaxime and ceftriaxone) were used more frequently, and ceftazidime and meropenem were used less frequently, than they would have been in empirical treatment based on the Japanese guidelines. Meropenem was used in only one case in which Gram-negative rods were observed despite cefotaxime administration.

Drug susceptibility tests of CSF cultures revealed that all antimicrobial treatments that were chosen based on PCGS results were effective against bacterial meningitis. All treatments that would have been chosen in the absence of PCGS findings were also effective.

The median dose (interquartile range) on the first day of admission for each antimicrobial chosen based on PCGS was as follows: ampicillin $8.5 \mathrm{~g}(8-12 \mathrm{~g})$, penicillin G 24 million units (24-24 million units), cefotaxime $9 \mathrm{~g}$ (8-12 g), ceftriaxone $4 \mathrm{~g}$ (4-4 g), vancomycin $2 \mathrm{~g}$ (2-2 g), meropenem $6 \mathrm{~g}(6-6 \mathrm{~g})$. The total cost on the first day of admission for all antimicrobials chosen based on PCGS was $78.5 \%$ of the total cost of all antimicrobials that would have been chosen under the Japanese guidelines (416,353 vs. 530,091 Japanese yen).

Table 4 Initial antibiotics selected based on PCGS versus those that would have been chosen under the Japanese guidelines

\begin{tabular}{|c|c|c|c|c|c|c|c|c|c|}
\hline & \multicolumn{4}{|c|}{ Point-of-care Gram stain $(\mathrm{N}=34)$} & \multicolumn{4}{|c|}{ Japanese guidelines $(\mathrm{N}=34)$} & \multirow[t]{3}{*}{$p$ value } \\
\hline & \multirow[t]{2}{*}{$\mathrm{GPC}(\mathrm{N}=23)$} & \multirow[t]{2}{*}{ GNR $(\mathrm{N}=8)$} & \multirow{2}{*}{$\begin{array}{l}\text { False- } \\
\text { negative } \\
(\mathrm{N}=3)\end{array}$} & \multirow[t]{2}{*}{ Total } & \multicolumn{2}{|c|}{ Immunocompetent } & \multirow{2}{*}{$\begin{array}{l}\text { Immunocompromised } \\
(\mathrm{N}=17)\end{array}$} & \multirow[t]{2}{*}{ Total } & \\
\hline & & & & & $\begin{array}{l}\text { Age }<50 \\
(N=7)\end{array}$ & $\begin{array}{l}\text { Age } \geq 50 \\
(N=10)\end{array}$ & & & \\
\hline Ampicillin/Penicillin G & $10 / 1$ & $4 / 0$ & $1 / 0$ & 16 & 0 & $5 / 0$ & $8 / 0$ & 13 & 0.62 \\
\hline Cefotaxime/Ceftriaxone & $17 / 6$ & $5 / 1$ & $3 / 0$ & 32 & 0 & $3 / 2$ & 0 & 5 & $<0.001^{*}$ \\
\hline Ceftazidime & 0 & 1 & 0 & 1 & 0 & 0 & 8 & 8 & $0.027^{*}$ \\
\hline Vancomycin & 18 & 1 & 2 & 21 & 0 & 10 & 17 & 27 & 0.18 \\
\hline Meropenem & 0 & 1 & 0 & 1 & 7 & 5 & 7 & 19 & $<0.001^{*}$ \\
\hline Clindamycin & 0 & 1 & 0 & 1 & 0 & 0 & 0 & 0 & 1.000 \\
\hline
\end{tabular}

GNR Gram-negative rod, GPC Gram-positive coccus, PCGS point-of-care Gram stain 
In aseptic meningitis, 39.2\% (38/97) of patients were treated with empiric antibiotics despite having negative PCGS findings (Table 1). This proportion was lower than that in a previous study on adult aseptic meningitis in which Gram staining was not available $(60.8 \%, 45 / 74)$ $(\mathrm{p}=0.006)[11]$.

\section{Discussion}

This observational study investigated the usefulness of PCGS in diagnosing and treating both bacterial and aseptic meningitis in adults. Our results yielded three main findings. First, PCGS was the most precise predictor for differentiating between bacterial and aseptic meningitis, being superior to medical histories, vital signs and physical examinations, and laboratory data available in the ER. Second, for bacterial meningitis, the selection of targeted therapies based on PCGS resulted in less frequent use of broad-spectrum antimicrobials compared with the simulated selection of empirical therapies based on the Japanese guidelines reported in the PGBM. Third, for aseptic meningitis, the selection of empiric therapies based on PCGS resulted in less frequent use of unnecessary antibiotics compared with a previous study in which Gram stain was not available.

Although most PCGS examinations in this study were performed by trainee doctors, the sensitivity and specificity of PCGS for detecting bacteria were $91.2 \%$ and $99.0 \%$ in bacterial and aseptic meningitis, respectively. Identification of bacterial strains based on PCGS was strongly correlated with that based on CSF culture results. These results are comparable to those of previous studies on Gram staining in microbiological laboratories [9, 12-14]. Accurate interpretation of CSF Gram stain results largely depends on the expertise of the individual performing the test [26]. PCGS can be interpreted with high accuracy by doctors who are experienced at performing and reading Gram stains, as studies on respiratory and urinary tract infections have shown [18, 27, 28]. At Okinawa Chubu Hospital, trainee doctors are still taught to consider Gram stain as part of a normal physical examination, as doctors in the United States used to be [23, 29, 30]. Their extensive experience with Gram stain and their eagerness to obtain PCGS results as soon as possible in order to initiate optimal antimicrobials enables them to perform and interpret PCGS with speed and accuracy.

In the sole false-positive case, a scant quantity of Gram-positive cocci was observed through PCGS, but this strain did not incubate in CSF culture. No previous antimicrobial agent had been administered in this case. Thus, debris or artifacts were suspected to have contaminated the Gram stain solution.

With regard to the three false-negative cases, one was L. monocytogenes, an intracellular pathogen for which
Gram staining offers a particularly low sensitivity of less than $50 \%[21,31]$. In the second case, the meningitis was a secondary infection site, while the primary infection focus was infective endocarditis or septic arthritis. The third case was an $H$. influenzae infection that had spread through CSF leakage. Previous studies have shown that the accuracy of Gram stain, that is, the correspondence between Gram staining and CSF culture results, depends strongly on bacterial load: Gram stain results were positive in only $25 \%$ of meningitis cases in which the CSF contained less than $10^{3} \mathrm{CFU} / \mathrm{ml}$, in $60 \%$ of cases with $10^{3}$ to $10^{5} \mathrm{CFU} / \mathrm{ml}$, and in $97 \%$ of cases with more than $10^{5} \mathrm{CFU} / \mathrm{ml}[10,32]$. Therefore, it is likely that, in each of our three false-negative cases, the bacterial load in the CSF was too small for PCGS to detect the bacteria. In spite of these cases' negative PCGS results, empirical antimicrobials were started in all three because bacterial meningitis was suspected due to low glucose level and high protein level in the CSF, strong suspicion of infectious endocarditis, and history of CSF leakage in the three cases, respectively.

In the one morphological mismatch case, PCGS seemed to indicate Gram-negative coccobacilli, but Gram stain in the laboratory revealed Gram-positive diplococci. After incubation, S. pneumoniae were identified through CSF culture, and the result of the Gram stain in the laboratory was correct. The reason of this mismatch could be that the crystal violet in the first stain was decolorized too much with acetone ethyl alcohol during the PCGS procedure and misidentified as Gram-negative. In the laboratory, Victoria blue was used for the first stain, and the blue was so vivid that it was easy to recognize Grampositive cases. An obvious mismatch between PCGS and laboratory-performed Gram stain was not found in the other cases we reviewed.

Compared with the simulated empirical therapies based on the Japanese guidelines reported in the PGBM, the targeted therapies based on PCGS were narrowerspectrum, equally effective, and 20\% lower in cost. PCGS enabled us not only to detect the presence of bacteria but also to identify their morphology. This permitted us to choose narrower-spectrum antimicrobials than the PGBM recommended, allowing us to keep pharmaceutical costs lower [18, 33]. If Gram-positive diplococcus was detected in PCGS, both third-generation cephalosporin and vancomycin were recommended because of the high prevalence of penicillin-resistant $S$. pneumoniae infection of the CSF in Japan [24]. The nationwide rate of S. pneumoniae resistance to penicillin-G in bacterial meningitis as assessed through CSF culture was 68.8\% (121/176) in 2001-2008 [34]. For pneumococcal meningitis, however, the co-administration of penicillin or meropenem was deemed unnecessary in the present clinical setting 
because the spectrum of third-generation cephalosporin with vancomycin sufficiently covers all of $S$. pneumoniae including the penicillin-resistant strains. If a Gram-negative rod was detected, vancomycin, which covers only Gram-positive species, was deemed unnecessary. The decision of whether to use carbapenem depended on local drug resistance patterns. In this population, meropenem was used in only one case, and ESBLs were not detected. It is expected, however, that meropenem use will increase in the future, given the ongoing increase in the incidence of ESBL-producing Enterobacteriaceae [24].

In aseptic meningitis, empiric antibiotics were used less frequently in our study, where PCGS findings were available, than they were in another study where Gram staining was only available during daytime hours [11]. The proportion of aseptic meningitis cases treated with empiric antimicrobials varies among institutions. A study in France reported using antimicrobials in only 35.2\% of aseptic meningitis cases (19/54), although this figure excludes cases with previous antimicrobial exposure [3]. A study in the USA, in contrast, reported using antimicrobials in $73.5 \%$ of aseptic meningitis cases (297/404), although this figure includes cases with previous antimicrobial exposure [35]. Another reason why it is difficult to compare our rate of antimicrobial use for aseptic meningitis with those in other regions is that the etiology of aseptic meningitis may vary among populations. However, the rate of empiric antimicrobial use after Gram stain results obtained later was $47.2 \%(35 / 74)$ [11], and it was similar to that at our institution $(p=0.28)$. Therefore, using PCGS anytime could help avoid the overuse of empiric antibiotics even for aseptic meningitis.

In bacterial meningitis cases, steroids were used in only $4 / 34$ cases (11.8\%). There were two reasons for this. One was that the study period was between 1995 and 2015. It was only in 2002 that the early use of dexamethasone for bacterial meningitis was proven to be effective, especially in patients with pneumococcal meningitis with GCS 8 to 11 [36]. The other was that some of our bacterial meningitis cases (10/34) were caused by S. stercoralis hyperinfection in this area [37], and steroid therapy is known to be harmful in some cases of strongyloidiasis [37]. Confirming the presence of Gram-negative rods or Grampositive cocci in chains encouraged us to search for $S$. stercoralis and to consider the possibility of disseminated strongyloidiasis.

This study has several limitations. First, some selection bias exists. The bacterial meningitis cases included only cases with positive CSF cultures. We excluded cases of meningitis related to surgical intervention, encephalitis, and probable aseptic meningitis with antimicrobial exposure in the previous $48 \mathrm{~h}$. HIV-infected patients were also excluded. In practice, however, hospitals will see many cases in which antimicrobials have already been administered, preventing clinicians from distinguishing between bacterial and aseptic meningitis. Second, this is a retrospective chart review study. We compared the accuracy of PCGS with that of Gram staining performed at a microbiological laboratory to the extent possible, though some old data were missing. Third, our results may not be directly applicable to other hospitals where PCGS is not regularly performed or where the prevalence of the various etiologic agents of meningitis is different. At most other hospitals, clinicians are unfamiliar with PCGS and lack the experience required to read Gram stain results [11]. Fourth, quality control of PCGS was not assessed in this study, although PCGS is the standard of care at Okinawa Chubu Hospital and its quality has been confirmed in several other studies $[18,27,28]$. Fifth, our ability to confirm the etiologies of aseptic meningitis was limited because further examinations other than those on the commercial level were not performed.

\section{Conclusions}

PCGS of CSF by clinicians is a valuable diagnostic tool for the discrimination of bacterial and aseptic meningitis in adults. PCGS enables the selection of narrower-spectrum antimicrobials for bacterial meningitis compared with the Japanese guidelines, and can potentially reduce the rate of empiric antimicrobial therapies for aseptic meningitis.

\section{Abbreviations \\ AUC: Area under the ROC curve; CSF: Cerebrospinal fluid; ER: Emergency room; ESBL: Extended-spectrum beta-lactamase; GCS: Glasgow Coma Scale; HIV: Human immunodeficiency virus; HTLV-1: Human T-lymphotropic virus-1; PCGS: Point-of-care Gram stain; PGBM: Practical Guideline for Bacterial Menin- gitis 2014; ROC: Receiver operating characteristic.}

\section{Acknowledgements}

We thank all trainee doctors at Okinawa Chubu Hospital $(\mathrm{OCH})$ who performed Gram staining in the ER; Mr. Noriyasu Kogachi, Mr. Teruyuki Hachiman, Ms. Mariko Teruya and all staff members of the Laboratory of Bacteriology at $\mathrm{OCH}$ for identifying bacterial pathogens; and Dr. Yoshihiko Raita of the Division of Nephrology at OCH for his review of this paper. This article is dedicated to Dr. Tomokazu Kishaba and Dr. Kazuo Endo, who were former attending physicians in the Division of Infectious Diseases at $\mathrm{OCH}$. Their educational guidance and emotional leadership for over 40 years enabled and still preserves the high quality of PCGS at this hospital. All of the authors of this paper have learned directly from them and are very grateful to them.

\section{Authors' contributions}

TT designed the protocol, collected and analyzed the data, and drafted the manuscript. ST, SS, and MN commented on previous versions of the manuscript and helped to draft the manuscript. All authors have read and approved the final manuscript.

Funding

None. 


\section{Availability of data and materials}

The datasets used and analyzed during the current study are available from the corresponding author on reasonable request.

\section{Ethics approval and consent to participate}

Because Gram-stain-based antimicrobial therapy is the standard practice at Okinawa Chubu Hospital, and because this was a retrospective observational study, written informed patient consent was deemed unnecessary. The study proposal was approved by the Institutional Review Board of Okinawa Chubu Hospital (No. 49, 2014)

\section{Consent for publication}

Not applicable.

\section{Competing interests}

The authors declare that they have no competing interests.

\section{Author details}

${ }^{1}$ Division of Infectious Diseases, Department of Internal Medicine, Okinawa Chubu Hospital, 281 Miyazato, Uruma, Okinawa 904-2293, Japan. ${ }^{2}$ Division of General Internal Medicine and Infectious Diseases, Hiroshima Prefectural Hospital, 1-5-54 Ujinakanda, Minamiku, Hiroshima 734-8530, Japan. ${ }^{3}$ Division of General Internal Medicine and Infectious Diseases, Sakibana Hospital, 1-3-30 Nozomino, Izumi, Osaka 594-1105, Japan.

Received: 14 October 2020 Accepted: 30 November 2020 Published online: 07 December 2020

\section{References}

1. McGill F, Heyderman RS, Panagiotou S, Tunkel AR, Solomon T. Acute bacterial meningitis in adults. Lancet. 2016;388(10063):3036-47. https:// doi.org/10.1016/s0140-6736(16)30654-7.

2. Proulx N, Frechette D, Toye B, Chan J, Kravcik S. Delays in the administration of antibiotics are associated with mortality from adult acute bacterial meningitis. QJM. 2005;98(4):291-8. https://doi.org/10.1093/qjmed/hci04 7.

3. Brivet FG, Ducuing S, Jacobs F, Chary I, Pompier R, Prat D, et al. Accuracy of clinical presentation for differentiating bacterial from viral meningitis in adults: a multivariate approach. Intensive Care Med. 2005;31(12):1654-60. https://doi.org/10.1007/s00134-005-2811-1.

4. Chen YY, Hsieh YC, Gong YN, Liao WC, Li SW, Chang IY, et al. Genomic insight into the spread of meropenem-resistant Streptococcus pneumoniae Spain(23F)-ST81, Taiwan. Emerg Infect Dis. 2020;26(4):711-20. https ://doi.org/10.3201/eid2604.190717.

5. Nakano S, Fujisawa T, Ito Y, Chang B, Matsumura Y, Yamamoto M, et al. Spread of Meropenem-Resistant Streptococcus pneumoniae Serotype 15A-ST63 Clone in Japan, 2012-2014. Emerg Infect Dis. 2018;24(2):275. https://doi.org/10.3201/eid2402.171268

6. Martin WJ. Rapid and reliable techniques for the laboratory detection of bacterial meningitis. Am J Med. 1983;75(1B):119-23.

7. McArthur R, Edlow JA, Nigrovic LE. Validation of the bacterial meningitis score in adults presenting to the ED with meningitis. Am J Emerg Med. 2016;34(7):1265-7. https://doi.org/10.1016/j.ajem.2016.04.003.

8. Tunkel AR, Hartman BJ, Kaplan SL, Kaufman BA, Roos KL, Scheld WM, et al. Practice guidelines for the management of bacterial meningitis. Clin Infect Dis. 2004;39(9):1267-84. https://doi.org/10.1086/425368.

9. Hasbun R, Bijlsma M, Brouwer MC, Khoury N, Hadi CM, van der Ende A, et al. Risk score for identifying adults with CSF pleocytosis and negative CSF Gram stain at low risk for an urgent treatable cause. J Infect. 2013;67(2):102-10. https://doi.org/10.1016/j.jinf.2013.04.002.

10. Greenlee JE. Approach to diagnosis of meningitis Cerebrospinal fluid evaluation. Infect Dis Clin North Am. 1990;4(4):583-98.

11. Tissot F, Prod'hom G, Manuel O, Greub G. Impact of round-the-clock CSF Gram stain on empirical therapy for suspected central nervous system infections. Eur J Clin Microbiol Infect Dis. 2015;34(9):1849-57. https://doi. org/10.1007/s10096-015-2423-9.

12. Karandanis D, Shulman JA. Recent survey of infectious meningitis in adults: review of laboratory findings in bacterial, tuberculous, and aseptic meningitis. South Med J. 1976;69(4):449-57.
13. Gaschignard J, Levy C, Romain O, Cohen R, Bingen E, Aujard Y, et al. Neonatal bacterial meningitis: 444 cases in 7 years. Pediatr Infect Dis J. 2011;30(3):212-7.

14. Tunkel AR, Scheld WM. Acute bacterial meningitis. Lancet. 1995;346(8991-8992):1675-80.

15. Lauer BA, Reller LB, Mirrett S. Comparison of acridine orange and Gram stains for detection of microorganisms in cerebrospinal fluid and other clinical specimens. J Clin Microbiol. 1981;14(2):201-5.

16. Neuman MI, Tolford S, Harper MB. Test characteristics and interpretation of cerebrospinal fluid gram stain in children. Pediatr Infect Dis J. 2008;27(4):309-13. https://doi.org/10.1097/INF.0b013e31815f53ba.

17. Wu HM, Cordeiro SM, Harcourt BH, Carvalho M, Azevedo J, Oliveira TQ, et al. Accuracy of real-time PCR, Gram stain and culture for Streptococcus pneumoniae, Neisseria meningitidis and Haemophilus influenzae meningitis diagnosis. BMC Infect Dis. 2013;13:26. https://doi. org/10.1186/1471-2334-13-26.

18. Taniguchi T, Tsuha S, Shiiki S, Narita M. Gram-stain-based antimicrobial selection reduces cost and overuse compared with Japanese guidelines. BMC Infect Dis. 2015;15:458. https://doi.org/10.1186/s12879-015-1203-6.

19. Taniguchi T, Tsuha S, Shiiki S, Narita M. High positivity of blood cultures obtained within two hours after shaking chills. International Journal of Infectious Diseases. 2018;76:23-8. https://doi.org/10.1016/j. ijid.2018.07.020.

20. Brouwer MC, Thwaites GE, Tunkel AR, van de Beek D. Dilemmas in the diagnosis of acute community-acquired bacterial meningitis. Lancet. 2012;380(9854):1684-92. https://doi.org/10.1016/s0140-6736(12)61185-4.

21. Gray LD, Fedorko DP. Laboratory diagnosis of bacterial meningitis. Clin Microbiol Rev. 1992;5(2):130-45.

22. Tapiainen T, Prevots R, Izurieta HS, Abramson J, Bilynsky R, Bonhoeffer J, et al. Aseptic meningitis: case definition and guidelines for collection, analysis and presentation of immunization safety data. Vaccine. 2007;25(31):5793-802. https://doi.org/10.1016/j.vaccine.2007.04.058.

23. Iwata K. Gram staining by physicians: an invaluable practice still seen in East Asia. Clin Infect Dis. 2004;39(11):1742-3. https://doi org/10.1086/425925.

24. Societas Neurologica Japonica JSoNT, Japanese Society for Neuroinfectious Diseases. Practical Guideline for Bacterial Meningitis. 2014. https:// www.neurology-jp.org/guidelinem/zuimaku_2014.html. Accessed 4 Dec 2020 .

25. Schlegel L, Grimont F, Ageron E, Grimont PA, Bouvet A. Reappraisal of the taxonomy of the Streptococcus bovis/Streptococcus equinus complex and related species: description of Streptococcus gallolyticus subsp. gallolyticus subsp. nov., S. gallolyticus subsp. macedonicus subsp. nov. and S. gallolyticus subsp. pasteurianus subsp. nov. Int J Syst Evol Microbiol. 2003;53(Pt 3):631-45. https://doi.org/10.1099/ijs.0.02361-0.

26. Brizzi K, Hines EM, McGowan KL, Shah SS. Diagnostic accuracy of cerebrospinal fluid gram stain in children with suspected bacterial meningitis. Pediatr Infect Dis J. 2012;31(2):195-7. https://doi.org/10.1097/INF.0b013 e31823d7b6f.

27. Fukuyama H, Yamashiro S, Kinjo K, Tamaki H, Kishaba T. Validation of sputum Gram stain for treatment of community-acquired pneumonia and healthcare-associated pneumonia: a prospective observational study. BMC Infect Dis. 2014;14:534. https://doi.org/10.1186/1471-2334-14-534

28. Yodoshi T, Matsushima M, Taniguchi T, Kinjo S. Utility of point-of-care Gram stain by physicians for urinary tract infection in children $\leq 36$ months. Medicine. 2019;98(14):e15101. https://doi.org/10.1097/md.00000 00000015101

29. Thomson RB Jr. One small step for the gram stain, one giant leap for clinical microbiology. J Clin Microbiol. 2016;54(6):1416-7. https://doi. org/10.1128/jcm.00303-16.

30. Provine $H$, Gardner P. The Gram-Stained Smear and Its Interpretation. Hospital Practice. 1974;9(10):85-91. https://doi.org/10.1080/21548 331.1974.11706891.

31. Brouwer MC, Tunkel AR, van de Beek D. Epidemiology, diagnosis, and antimicrobial treatment of acute bacterial meningitis. Clin Microbiol Rev. 2010;23(3):467-92. https://doi.org/10.1128/cmr.00070-09.

32. La Scolea $L J$, Dryja D. Quantitation of bacteria in cerebrospinal fluid and blood of children with meningitis and its diagnostic significance. J Clin Microbiol. 1984;19(2):187-90.

33. Kishaba T. How to use antibiotics in major categories of infectious diseases_-bacterial meningitis (In Japanese). Medicina. 1999;36:102-3. 
34. Shinjoh M, Iwata S, Yagihashi T, Sato Y, Akita H, Takahashi T, et al. Recent trends in pediatric bacterial meningitis in Japan-a country where Haemophilus influenzae type B and Streptococcus pneumoniae conjugated vaccines have just been introduced. J Infect Chemother. 2014;20(8):47783. https://doi.org/10.1016/j.jiac.2014.04.007.

35. Shukla B, Aguilera EA, Salazar L, Wootton SH, Kaewpoowat Q, Hasbun R. Aseptic meningitis in adults and children: diagnostic and management challenges. J Clin Virol . 2017;94:110-4. https://doi.org/10.1016/j. jcv.2017.07.016.

36. de Gans J, van de Beek D. Dexamethasone in adults with bacterial meningitis. N Engl J Med. 2002;347(20):1549-56. https://doi.org/10.1056/NEJMo a021334.
37. Sasaki Y, Taniguchi T, Kinjo M, McGill RL, McGill AT, Tsuha S, et al. Meningitis associated with strongyloidiasis in an area endemic for strongyloidiasis and human T-lymphotropic virus-1: a single-center experience in Japan between 1990 and 2010. Infection. 2013;41(6):1189-93. https://doi. org/10.1007/s15010-013-0483-2.

\section{Publisher's Note}

Springer Nature remains neutral with regard to jurisdictional claims in published maps and institutional affiliations.
Ready to submit your research? Choose BMC and benefit from:

- fast, convenient online submission

- thorough peer review by experienced researchers in your field

- rapid publication on acceptance

- support for research data, including large and complex data types

- gold Open Access which fosters wider collaboration and increased citations

- maximum visibility for your research: over $100 \mathrm{M}$ website views per year

At BMC, research is always in progress.

Learn more biomedcentral.com/submissions 